\title{
REVENDO ESTUDOS SOBRE A ASSISTÊNCIA DOMICILIAR AO IDOSO
}

\author{
Andreza Aparecida de Lima ${ }^{1}$ \\ Regina Stella Spagnuolo \\ Karina Pavão Patrício \\ Universidade Estadual Paulista Júlio de Mesquita Filho, Botucatu-SP, Brasil
}

\begin{abstract}
RESUMO. A assistência domiciliar apresenta-se como uma recente modalidade de cuidado aos idosos, sobretudo no Brasil, e vem sendo inserida nas políticas públicas. Este estudo busca identificar produções científicas acerca da assistência domiciliar, a fim de verificar quais as contribuições desta na dinâmica social do idoso. A pesquisa foi realizada nas bases de dados LILACS e MEDLINE, com artigos publicados entre os anos de 2001 a 2011, nos idiomas português e inglês. A combinação de palavras-chave utilizada foi home care e elderly, elderly e care, e elderly e caregiver, e as correspondentes em português. A discussão dos resultados evidenciou a importância da assistência domiciliar, principalmente para idosos solitários ou negligenciados. A revisão revelou poucos estudos referentes à assistência domiciliar na dinâmica social de idosos e a necessidade de ampliar as discussões para além do campo da saúde.
\end{abstract}

Palavras-chave: Assistência domiciliar; velhice; saúde.

\section{REVIEWING STUDIES ON HOME CARE FOR THE ELDERLY}

\begin{abstract}
Home care presents itself as a new modality of care for the elderly, especially in Brazil, and has been inserted into public policies. This study aims to identify scientific productions about home care, in order to ascertain which are the contributions of this social dynamic in the elderly. The research was conducted in the databases MEDLINE and LILACS, with articles published between the years 2001-2011, in Portuguese and English. The combination of keywords used was home care and elderly , elderly and care, and elderly and caregiver, and correspondents in Portuguese. The discussion highlighted the importance of home care, especially for lonely or neglected elderly. The review revealed few studies concerning the social dynamics in the home care of the elderly and the need to broaden the discussion beyond the field of health.
\end{abstract}

Keywords: Home care; old age; health.

\section{REVISIÓN DE ESTUDIOS SOBRE LA ATENCIÓN DOMICILIARIA A LAS PERSONAS MAYORES}

RESUMEN. La atención domiciliaria se presenta como una nueva modalidad de atención a los ancianos, especialmente en Brasil, y se ha insertado en las políticas públicas. Este estudio tiene como objetivo identificar producciones científicas acerca de la atención domiciliaria, a fin de determinar cuáles son las aportaciones de esta dinámica social de las personas mayores. La investigación se realizó en la bases de datos MEDLINE y LILACS, con artículos publicados en 2011 en Portugués e Inglés. La combinación de palabras clave se utilizó y el cuidado de asilo de ancianos, de edad avanzada y la atención, y las personas mayores y cuidadores, así como corresponsales en portugués. El debate puso de relieve la importancia de los cuidados en el hogar, especialmente para los ancianos solos o abandonados. El examen reveló pocos estudios sobre la dinámica social en la atención domiciliaria de las personas mayores y la necesidad de ampliar el debate más allá del campo de la salud.

Palabras-clave: Atención domiciliaria de salud; vejez; salud.

Nos últimos anos o envelhecimento tornou-se uma questão social muito discutida e pesquisada em diversos países do mundo. O tempo de vida das pessoas tem aumentado consideravelmente (Veras, 2009), e essa nova realidade traz ao convívio social um maior número de pessoas que

\footnotetext{
${ }^{1}$ Endereço para correspondência: Al. Manoel Figueiredo, 5-17, Pq. São Geraldo. CEP 17.021-310 - Bauru-SP, Brasil. E-mail: drelimapsico@yahoo.com.br
} 
esperam viver mais, e com melhor qualidade de vida e bem-estar.

Não obstante, o processo de envelhecimento, além das alterações biológicas e físicas, que são mais visíveis, traz também alterações psíquicas e sociais. Essas alterações podem desencadear a necessidade de transformações relacionadas à aceitação ou não deste processo de envelhecimento e dos valores e interesses assimilados ao longo da vida (Doll, 2006).

Essa é uma fase de perdas: perda de salário em relação à aposentadoria, perda de status social, perda de familiares ou colegas idosos, perdas na saúde - enfim, para eles o passado representa a vida e o presente a morte (Patrício, 2009).

Para enfrentar esta fase, muitos idosos necessitam de apoio e cuidado. Estes cuidados, muitas vezes, provêm da ajuda familiar, de amigos ou da comunidade onde o idoso vive, reforçando a necessidade de fortalecer as redes de apoio familiar e social diante do acelerado envelhecimento populacional (Resende, Cunha, Silva \& Souza, 2007); porém muitos idosos não têm este apoio social e também não conseguem substituir esta ausência por um cuidado especializado, em vista da dificuldade de acesso aos serviços disponíveis ou, por serem muito onerosos, aos serviços particulares.

Conforme Silva e Fonseca (2007), a formação dos sistemas de proteção e apoio social tem origem nas necessidades das sociedades de impedir ou diminuir o impacto de determinados riscos sobre os indivíduos ou grupos sociais.

Siqueira (2008) ressalta que o apoio social é um conceito ainda em construção. É qualquer informação - falada ou não - e/ou auxílio material fornecido por grupos e/ou pessoas que se conhecem que resultem em efeitos emocionais e/ou comportamentos positivos. Por ser um processo recíproco, focaliza a qualidade das interações e deve gerar efeitos positivos tanto para quem recebe quanto para quem oferece apoio.

Nos últimos anos, particularmente nas sociedades ocidentais, a temática do apoio social tem adquirido crescente relevância e, a partir dos anos 70, passou a constituir um quadro teórico integrado e consistente, assumindo-se como um processo promotor de assistência e ajuda por meio de fatores de suporte que facilitam e asseguram a sobrevivência dos seres humanos (Silva \& Fonseca, 2007).

Rodrigues e Seidl (2008) ressaltam que nesta época pesquisadores começaram a questionar a possibilidade de o apoio social constituir um fator de proteção às pessoas em uma variedade de crises e doenças, desde o baixo peso ao nascer até a morte.

Desde os primórdios, a assistência à saúde era realizada no domicílio, do paciente ou do próprio médico. Imhotep talvez seja um dos primeiros médicos famosos, no século XIII antes de Cristo, a possuir um local próprio para atender os clientes, um misto de consultório e hospital, com atendimentos por meio de visitas domiciliares. $\mathrm{Na}$ Grécia antiga, o médico Asklépios atendia os doentes in loco, e Samuel Hanneman, fundador da Homeopatia, acreditava que o médico era responsável por recrutar os enfermos e cuidar deles em suas casas (Sarton, 1998).

Nos âmbitos da América do Norte e da Europa, a assistência domiciliar, como forma organizada de cuidado, surgiu nos Estados Unidos em 1947 e teve como motivação inicial descongestionar os hospitais e proporcionar aos pacientes e familiares um ambiente psicossocial mais favorável. No Canadá, os serviços de assistência domiciliar funcionam desde os anos 60 e eram orientados para a alta precoce de pacientes cirúrgicos.

O crescimento da assistência domiciliar no Brasil é recente. Duarte e Diogo (2000) citam que o desenvolvimento do atendimento domiciliar no país, inicialmente, manteve maior ligação com o serviço de enfermagem, quando em 1919 criou-se o Serviço de Enfermeiras Visitadoras no Rio de Janeiro, com o objetivo de promover a educação em saúde da população em suas casas.

A implantação da assistência domiciliar como uma atividade planejada teve início há quase quarenta anos no Hospital do Servidor Público Estadual de São Paulo, cujo foco era desospitalizar doentes crônicos estáveis para desocupar uma parte dos leitos superlotados.

Os serviços de assistência domiciliar no Brasil estiveram, por muito tempo, ligados à área de saúde pública (Duarte \& Diogo, 2000), e o cuidado domiciliar aparece como uma alternativa para evitar ou abreviar internações hospitalares, reduzindo custos (Feuerwerker \& Merhy, 2008). Além disso, pode promover o estímulo e a manutenção da autonomia do paciente, pois as tarefas no domicílio podem ser executadas no tempo que convenha ao paciente, com o incentivo, apoio e possibilidade de maior desenvolvimento do vínculo entre o cuidador e o doente (Brondani, 2008; Feuerwerker \& Merhy, 2008). 
A assistência domiciliar é uma das mais recentes modalidades de cuidado, sobretudo no Brasil (Lacerda, Giacomozzi, Oliniski \& Truppel, 2006; Schwonke, Silva, Casalinho, Santos \& Vieira, 2008), e visa à promoção, manutenção e/ou restauração da saúde e o desenvolvimento e adaptação de suas funções de maneira a favorecer o restabelecimento de sua independência e a preservação de sua autonomia (Duarte \& Diogo, 2000).

Duarte e Diogo (2000) ressaltam que a assistência domiciliar é uma opção segura e eficiente para $o$ atendimento a determinados tipos de paciente que, mesmo após a estabilização do quadro clínico, continuam necessitando de cuidados no cotidiano.

Para Feuerwerker e Merhy (2008), a assistência domiciliar amplia a autonomia dos usuários e de suas famílias e, apesar de ser uma alternativa, a demanda por este tipo de serviço é maior do que a oferta.

Diante do grande e acelerado envelhecimento populacional, a assistência domiciliar ao idoso torna-se uma ferramenta fundamental visando diminuir possíveis riscos fisiológicos, imunológicos, emocionais e sociais, além de reintegrar o idoso em seu núcleo familiar e de apoio, resgatando sua identidade e o pertencimento social.

O despertar da área governamental e do setor privado para a questão do idoso surgiu no início dos anos 1970 (Veras, 2009). Em 1994 criou-se a Política Nacional do Idoso (Brasil, 1994), cujo objetivo é assegurar seus direitos sociais, criando condições para promover sua autonomia, integração e participação efetiva na sociedade. A Política Nacional de Saúde da Pessoa Idosa (Brasil, 2006) foi aprovada no ano de 2006 e assegura a implantação de serviços de atenção domiciliar.

Como o Brasil está em processo de envelhecimento, o número de idosos é elevado. Em vista desta grande demanda, os serviços destinados a atender ou acolher adequadamente estes idosos é pequeno, o que os leva recorrer a serviços privados, quando podem arcar com os custos ou contar com o apoio de movimentos sociais como as igrejas, ONGs e o voluntariado.

Estes serviços de assistência domiciliar voltados para a dinâmica social no Brasil vão desde cuidados de atividades de vida diárias até suporte comunitário, realização de tarefas externas, como, ida a um banco, supermercado ou a uma farmácia, além de fazerem aumentar a frequência de contatos e intercâmbios sociais, trazendo à tona aspectos subjetivos do sujeito, fatores que resultam em melhora e proteção da saúde (Guedea, Albuquerque, Troccóli, Noriega, Seabra \& Guedea, 2006).

Neste sentido objetivou-se apresentar uma revisão da literatura sobre a assistência domiciliar ao idoso, investigando as discussões sobre a dinâmica social do idoso nestas publicações.

\section{MÉTODO}

Partiu-se de uma revisão de literatura para identificar produções sobre a temática da assistência domiciliar. Adotou-se a revisão integrativa da literatura, que contribui para 0 processo de sistematização e análise dos resultados, visando à compreensão de determinado tema a partir de outros estudos independentes.

Este tipo de revisão sumariza pesquisas passadas e tira conclusões globais de um corpo de literatura em particular, permitindo a construção de uma análise ampla e assim contribuindo para discussões sobre métodos e resultados de pesquisa e para reflexões sobre a realização de estudos futuros (Ganong, 1987).

Para tanto, foram seguidas as seguintes etapas: seleção da pergunta de pesquisa; definição dos critérios de inclusão de estudos e seleção da amostra; análise crítica dos achados; interpretação dos resultados e considerações sobre a evidência encontrada, discutidos a seguir.

Considerando a assistência domiciliar sob outros aspectos e as influências sofridas pela política de saúde na construção destas novas vertentes, esta revisão pretendeu responder à seguinte questão: "Quais têm sido as contribuições da assistência domiciliar para o envelhecimento considerando a dinâmica social atual?".

As buscas foram realizadas nas bases de dados LILACS e MEDLINE, através de acesso on line, considerando o período de publicação entre os anos de 2001 a 2011. A combinação de palavraschave utilizadas no levantamento de literatura foi home care e elderly, care e elderly, caregiver e elderly, e as correspondentes na língua portuguesa.

Os dados obtidos foram organizados através de fichamentos e a representação das características foi feita por meio de tabelas, para melhor visualização de uma quantidade expressiva 
de dados com vistas a facilitar sua organização e o estabelecimento das categorias de análise.

Como critérios de inclusão foram adotados trabalhos na íntegra, nos idiomas português e inglês. Foram excluídos trabalhos como monografias, dissertações, teses, livros, capítulos e resenhas de livros, manuais, relatórios técnicos e científicos, artigos de revisão e artigos incompletos ou resumos não disponíveis on line em outras línguas e que não se relacionassem com a temática da assistência domiciliar na dinâmica social.

O processo de análise deu-se na vertente qualitativa, que se utilizou a análise de conteúdo do tipo análise temática para organizar o conhecimento produzido em categorias desdobradas em três fases: pré-análise; exploração do material; tratamentos dos resultados, com inferência e interpretação (Bardin, 2011).

Foram extraídos os principais aspectos abordados em cada artigo, os quais foram comparados e agrupados por similaridade de conteúdo, sob a forma de categorias empíricas, sendo construídas três categorias para análise, assim especificadas: vantagens da assistência domiciliar; o acesso dos idosos aos serviços de saúde e prevenção; e, despreparo dos cuidadores familiares e dos serviços de saúde.

\section{RESULTADOS E DISCUSSÃO}

Por meio da pesquisa por assunto, utilizandose inicialmente a combinação das palavras-chave "home care" e "elderly" e as correspondentes na língua portuguesa, foram encontrados 206 artigos.

Em uma segunda busca, utilizando-se a combinação "elderly" com as palavras-chave "care" e "caregiver" e as correspondentes na língua portuguesa, foram encontrados 144 e 67 artigos, respectivamente.

Como o foco do presente estudo era a assistência domiciliar e suas contribuições na dinâmica social do idoso, após esta busca criteriosa restaram somente oito artigos, sendo quatro indexados na base de dados MEDLINE e quatro na LILACS.

Inicialmente chama a atenção a escassez de trabalhos recentes publicados sobre a temática investigada, não obstante o intenso envelhecimento populacional e a necessidade crescente de assistência domiciliar voltada aos idosos.

Notou-se que existe uma produção majoritária de publicações em revistas internacionais (87.5\%).
Quanto ao idioma, o predominante foi o inglês (75\%), sendo apenas dois artigos escritos no idioma português. Quanto ao local de publicação dos artigos da amostra, verificou-se que a maior parte das publicações é brasileira, correspondendo a $37.5 \%$ da amostra, com três artigos publicados, seguida pelas de países como Canadá, a Escócia, a França, o Japão e a Nigéria, com a publicação de um artigo cada, correspondendo a $12.5 \%$.

$O$ método quantitativo foi predominante em $62,5 \%$ das publicações, porém sabe-se que o método qualitativo é importante para estudos deste tipo, por possibilitar compreensões mais aprofundadas, associadas com sentimentos e sensações, muito importantes quando se trabalha no campo da assistência domiciliar a idosos.

Entre os autores que mais publicaram estão os médicos $(37.5 \%)$ e enfermeiros $(37.5 \%)$, sendo que a totalidade dos profissionais está vinculada a universidades e instituições de ensino, tendo como titulação mínima a de doutor. Os dados mostram que as publicações neste campo estão concentradas nas academias.

Nota-se que os serviços deveriam ser estimulados e apoiados a publicar suas experiências, que neste campo da assistência domiciliar atuam muito na atenção primária à saúde, principalmente nos serviços de saúde da família. São estes serviços os principais responsáveis por despertar estas novas particularidades dentro da assistência domiciliar, em relação ao trabalho desempenhado principalmente pelos agentes comunitários de saúde em suas visitas domiciliares de rotina, e por trazer à tona as contribuições aos que fazem uso dessa assistência.

A leitura do corpus de análise permitiu sistematizar o conhecimento produzido, em três temas: Vantagens da assistência domiciliar; $O$ acesso dos idosos aos serviços de saúde e prevenção; e Despreparo dos cuidadores familiares e dos serviços de saúde.

\section{Vantagens da assistência domiciliar}

Dos artigos encontrados, $37.5 \%$ se referiam a esta temática. $\mathrm{A}$ análise desses artigos evidencia que tem havido um desenvolvimento desigual da assistência domiciliar ao longo do tempo.

Os artigos ressaltam que a satisfação do paciente tem sido um assunto amplamente investigado em pesquisas de saúde, enquanto a qualidade dos cuidados a partir da perspectiva do paciente, especialmente nos cuidados de saúde 
em casa, assou a ser investigada apenas muito recentemente (Pérodeau, Paradis, Grenier, O'Connor e Grenon, 2011; Hayashi, Nomura, Ina, Kato, Hirose, Nonogaki \& Suzuki, 2011; BradburyJones, Irvine, Jones, Kakehashi \& Ogi, 2011).

Segundo Duayer e Oliveira (2005), a doença neste cenário é o vínculo entre o paciente, a família e os profissionais da saúde, e quanto maior o empenho baseado no respeito, no amparo, no diálogo e no afeto por parte destes, maior també é a viabilização da recuperação do paciente, acelerando o seu processo de melhora.

Os autores Pérodeau et al. (2011) atentam para os cuidados domiciliares a idosas usuárias de substâncias psicotrópicas no Canadá, principalmente àquelas com sintomas de ansiedade e insônia. Seu estudo descreve o benefício dos cuidados em domícilio após tratamento específico e a correlação desta assistência com a melhora da autoimagem das idosas. Os resultados trouxeram à tona os efeitos psicossociais que a assistência domiciliar pode trazer, aliando os benefícios não só do usuário, mas também de seu cuidador, que lança mão de novas estratégias de cuidado e conta com outras fontes de apoio social.

As percepções positivas do adoecimento surpreenderam. A elevação da autoestima, já que o adoecimento, de modo geral, abalou a integridade corporal e muitas vezes estética, foi vista como uma experiência que aproximou mais o sujeito dele mesmo, não só pelo aumento da ociosidade, mas, principalmente, pela mudança abrupta em seu estilo de vida e na maneira de percebê-la, assim como no modo de olhar a si mesmo, que pode ser uma vivência de crescimento pessoal.

Em outro estudo, foram investigados 252 idosos japoneses e os fatores associados ao local de morte de pacientes que recebem serviços de assistência domiciliar, e, segundo esse estudo, 40 pacientes morreram em casa e 59 pacientes morreram em hospitais (Hayashi et al., 2011).

Os pacientes que morreram em casa receberam um prazo mais curto de atendimento domiciliar, sofreram neoplasias mais frequentemente e receberam serviços de enfermagem com mais frequência do que os que morreram nos hospitais. Aqueles que morreram em casa apresentaram uma maior probabilidade de sofrer de doença malígna em comparação com os idosos que morreram em hospitais.

Há poucas pesquisas relacionadas aos estágios finais de vida nas quais a residência tenha sido o local de morte. Sabe-se apenas que os familiares de quem morreu em hospitais correm mais riscos de sofrer distúrbios psiquiátricos e que as pessoas responsáveis pelos cuidados destes pacientes têm cinco vezes mais riscos de desenvolver estresse pós-traumático do que aquelas que acompanham os últimos dias desses pacientes em casa (Hayashi et al., 2011).

Segundo os autores, não só os pacientes que morrem em hospitais têm pior qualidade de vida em seus últimos dias, mas também os familiares e amigos que o acompanham até a morte acabam apresentando mais danos físicos e psicológicos. Em casa, os pacientes recebem os cuidados que aliviam e confortam, permitindo uma morte com menos sofrimento para todos.

Em um terceiro estudo foram analisadas as experiências de enfermeiros que cuidam de pessoas idosas no Reino Unido e no Japão (Bradbury-Jones et al., 2011). Os dados foram analisados por meio de uma análise temática contando com o recurso de autorrelatos. A análise revelou semelhanças nas experiências de enfermeiros de ambos os países. Em todos os autorrelatos apareceu a questão de que são muitas as recompensas encontradas ao se trabalhar com pessoas mais velhas e que há uma relação de troca estabelecida, em que cuidar vai além de afastar o idoso da doença; ouvir suas histórias de outros tempos e fazer companhia, por exemplo, são momentos preciosos do trabalho.

$\mathrm{Na}$ totalidade dos estudos, percebe-se que a assistência domiciliar pode ser benéfica tanto para os pacientes como para os familiares ou amigos que o acompanham. Além disto, pode trazer benefícios dentro da própria dinâmica do serviço de saúde, como diminuição das internações hospitalares, aumento do contato familiar, atendimento mais humanizado, comodidade e conforto, fácil acesso ao profissional, segurança no atendimento e facilidade na realização de exames diagnósticos.

Em determinados casos de patologias mais severas, que apresentem a necessidade de um cuidado mais integral e especializado, a assistência domiciliar sofre a falta de estrutura adequada e do auxílio de uma gama diversificada de profissionais da saúde, o que acentua dificuldades como o aumento dos custos, despesas com cuidador, adequação do domicílio e indisponibilidade da família.

Dentre os artigos analisados, a maioria teve seu foco nos aspectos vinculados aos serviços de 
saúde, discutindo superficialmente a dinâmica social do idoso. Evidencia-se a necessidade de estudos com abordagens mais amplas sobre as particularidades da assistência domiciliar no contexto social.

\section{O acesso dos idosos aos serviços de saúde e prevenção}

Dos artigos encontrados e analisados, 37.5\% discutiam o acesso aos serviços e a prevenção.

O primeiro estudo foi realizado na França, e investigou as relações entre a procura por serviços odontológicos e os fatores socioeconômicos e demográficos dos idosos (Lupi-Pegurier, ClercUrmes, Abu-Zaineh, Paraponaris \& Ventelou, 2011).

Os idosos de baixa renda e sem plano de saúde complementar foram asssociados a maior chance de nunca ter visitado um dentista, o que revela a falta de acesso à assistência odontológica. O plano de saúde complementar apareceu como um fator significativo para o acesso aos serviços odontológicos nesse país.

O segundo estudo pretendeu examinar as causas e o padrão de morte em idosos, relacionado ao acesso destes aos serviços de saúde (Sanya, Abiodun, Kolo, Olanrewaju \& Adekeye, 2011). Os autores pesquisaram pacientes com idade acima de 60 anos gerenciados em um hospital terciário na Nigéria, com escassos registros de mortalidade, entre janeiro de 2005 e junho de 2007. As informações incluíram dados demográficos e a duração da admissão e diagnóstico. As causas de morte foram determinadas a partir de notas de evolução clínica e diagnóstico.

Ocorreram 297 mortes em pessoas com mais de 60 anos de idade, cuja taxa de mortalidade representou $22,8 \%$. Os homens foram os que mais morreram. As mulheres morreram mais velhas, com aproximadamente 69.7 anos. Os diagnósticos mais comuns nas mortes foram acidente vascular cerebral, doenças infecciosas e doenças do trato respiratório.

Os autores salientam que, por um grande número de idosos pertencerem à camada de baixa renda da população, muitos vivem em locais inadequados e com infraestrutura insuficiente, condições em que é precária a higiene, tanto a pessoal quanto a coletiva. Falta de informação, hábitos culturais indesejáveis e falta de recursos financeiros podem contribuir para que esses riscos aumentem, dificultando a prevenção e o prognóstico de doenças que acometem essa faixa etária da população.

Com relação a quem morre mais - se adultos, idosos ou muito idosos -, os autores Oliveira, Nogueira, Andolhe, Padilha e Sousa (2011) realizaram um estudo, comparando a evolução clínica dessas faixas etárias em pacientes internados em unidades de terapia intensiva (UTIs) localizadas na cidade de São Paulo/Brasil.

Entre adultos e idosos ocorreu diferença em relação à mortalidade, com maior taxa no grupo mais velho; entretanto a mortalidade dos muito idosos e adultos foi similar. Em geral, os resultados indicaram que a idade mais avançada não foi fator associado aos desfechos indesejáveis da assistência intensiva.

Isto significa que, qualquer que seja o indicador de saúde analisado, os resultados mostram maior proporção de agravos nos indivíduos com mais de 60 anos, decorrentes de doenças crônicas, os quais devem ser precocemente identificados, tratados e monitorados, com vista a reduzir a mortalidade e os gastos com a saúde. Para os autores, o envelhecimento da população mundial oferece desafios médicos e socioeconômicos aos órgãos governamentais e à sociedade. (Oliveira et al., 2011).

Quanto mais efetivo o trabalho de prevenção, mais idosos poderão ser cuidados em seu lar, o que faz diminuírem os gastos e permite mantê-los em um ambiente confortável e seguro, reduzindo as internações e reinternações hospitalares.

Assim, questões como a renda, a escolaridade, o sexo do paciente e a morbidade referida podem não estar associadas à necessidade de acesso, mas a uma baixa capacidade de utilização de serviços de saúde pelos idosos.

As desigualdades socioeconômicas levam a diferentes tempos e formas de adoecer e diferentes necessidades e capacidades de acesso aos serviços de saúde. Idosos com pior escolaridade apresentam pior estado de saúde, em função de piores hábitos, maior exclusão e menor nível de informação e condições socioeconômicas para acessar serviços de saúde precocemente.

\section{Despreparo dos cuidadores familiares e dos serviços de saúde}

Nesta revisão foram encontrados e analisados $25 \%$ dos artigos com a temática dos cuidadores e o perfil destes quando o assunto é a assistência domiciliar. 
O cuidar, no cenário domiciliar, é uma experiência ligada ao ser humano presente desde sempre na história da humanidade. Diante da doença, o cuidador assume a responsabilidade de cuidar, no entanto, na maioria das vezes não recebeu nenhum tipo de preparo para esta situação, adquirindo experiência pela tentativa e erros, pela repetição ou orientações informais da comunidade adjacente. Isto transforma 0 ato de cuidar em domicílio em um aprendizado contínuo e diário (Brondani, 2008).

Dentro da rede social dos idosos, a família tem sido apontada como a fonte primária de suporte, formando, juntamente com os vizinhos e amigos, uma rede informal (Resende et al., 2007).

Em geral a cuidadora é a esposa e, em segundo lugar, numa hierarquia de compromisso, a filha mais velha. As mulheres costumam assumir os cuidados mesmo em famílias com filhos homens, os quais participam com ajuda material e tarefas externas, como deslocar o paciente para outros ambientes ou custear medicamentos e intervenções laboratoriais e hospitalares (Beger, 2008).

Ainda, segundo os artigos, é comum que a mulher assuma os cuidados mesmo que trabalhe fora, o que acaba tendo como consequência uma diminuição das atividades de lazer e vida social desta cuidadora.

O primeiro estudo analisou o discurso de acompanhantes de Campina Grande, na Paraíba Brasil, com relação ao idoso hospitalizado, buscando meios para facilitar o processo de cuidados tanto no período de internação como após a alta hospitalar (Medeiros, Nóbrega, Sousa \& Silva, 2011). Os resultados apontam que há uma necessidade de preparação da equipe hospitalar que atende ao idoso, no que se refere a um olhar direcionado e diferenciado aos cuidadores, para a garantia da continuidade da assistência no período pós-alta hospitalar.

Os cuidadores relatam que se veem perdidos ao dar continuidade ao tratamento oferecido no hospital, com relação tanto à falta de informação quanto aos sentimentos de insegurança e medo. Isso faz com que o cuidador familiar nem perceba quão significativo é, para o idoso, estar em sua casa e se restabelecer neste ambiente, que the é mais seguro, conhecido e confortável. Muitas vezes o cuidador julga que não está fazendo o mais certo, que seu idoso deveria voltar ao hospital, que ele não consegue cuidar adequadamente sem uma sobrecarga imensa. Isto reforça a falta de apoio e orientação aos cuidadores de idosos nos serviços de saúde.

Muitas vezes a equipe de saúde dos hospitais acaba não prestando ao idoso um atendimento mais adequado e humanizado, sem levar em conta a pessoa em sua totalidade, devido à demanda, à falta de recursos e à formação mecanicista, além da desmotivação por conta dos baixos salários.

Assim, os cuidadores familiares, muitas vezes sem preparo e conhecimento ou senso crítico, acabam não questionando os cuidados recebidos pelo idoso nos serviços de saúde, ficando receosos em perguntar para esclarecer dúvidas em relação aos cuidados que devem ter em casa com o idoso antes da alta hospitalar de seus ente.

A Universidade Federal de São Carlos (UFSCAR) realizou um estudo, por meio de médicos do Departamento de Medicina, com o objetivo de identificar os fatores independentes que sobrecarregam os cuidadores na cidade de São Paulo/Brasil (Tamanini, Santos, Lebrão, Duarte \& Laurenti, 2011). Segundo os autores, alguns doentes idosos com incontinência urinária requerem cuidado de terceiros. Tal cuidado pode ocorrer diariamente, deixando poucas oportunidades para o cuidador cuidar de si mesmo. A prevalência geral de incontinência urinária foi maior entre as mulheres. Houve associação significativa positiva entre sobrecarga do cuidador e pacientes incontinentes, demonstrando que a incontinência urinária e a disfunção cognitiva em pacientes idosos produzem maior sobrecarga do cuidador.

O fardo de cuidar não está associado apenas às alterações de comportamento do paciente, mas também à sua dificuldade em realizar tarefas rotineiras e à insatisfação do cuidador com a assistência de parentes e amigos. Assim, não há necessidade de atuar de forma complexa quando se tenta solucionar ou amenizar os problemas encontrados, mas muitas vezes se agi muito tecnicamente, sem perceber que o que realmente importa é o olhar, o sentir, o tocar e o solidarizar-se.

Uma vez que o cuidado envolve zelo, preocupação e outros elementos subjetivos, a dedicação, o bom senso e a superação do medo e da insegurança serão fatos que permearão constantemente o cotidiano do cuidador.

\section{CONSIDERAÇÕES FINAIS}

De modo geral, nesta revisão integrativa da literatura sobre assistência domiciliar aos idosos, 
notou-se que os estudos giram em torno de discussões na esfera do adoecimento físico, dos serviços e falta de capacitação dos familiares, ou seja, concentram-se em torno de um eixo central que é a saúde física do idoso. O papel da assistência domiciliar na dinâmica social do idoso é abordado apenas superficialmente.

$O$ fato é que vemos o envelhecimento populacional se alastrando por todo o país e, por outro lado, os serviços de saúde se adequando a esta realidade, assim como os profissionais sendo melhor preparados; no entanto existe um grande descompasso nestes dois processos, pois 0 envelhecimento caminha a grandes passadas, envolvendo aspectos transdisciplinares e multicausais, enquanto o preparo dos serviços é muito lento e unicausal, enfocando quase exclusivamente os aspectos físicos, sendo os sociais e psicológicos muitas vezes encaminhados aos especialistas, não sendo estas discussões incorporadas ao cuidado diário.

$E$ neste meio que aparece a figura fundamental do cuidador familiar, que, não obstante, na escala aparece por último, não sendo capacitado, acolhido e nem mesmo ouvido pelos serviços. Isto gera um circulo vicioso no qual o cuidador acredita que não sabe ou não cuida adequadamente, buscando mais internações hospitalares, que oneram 0 serviço e trazem mais sofrimento aos idosos e também aos familiares, que, diante disto, têm que levar de volta o idoso para casa, mas, pela insegurança frente à falta de orientação, voltam a questionar se estão fazendo 0 melhor, perpetuando, assim, o ciclo da baixa qualidade de vida e das infelicidades em relação ao envelhecimento doente.

A relação dos idosos com os serviços de saúde é intensa, podendo traduzir-se em injustiças e inadequações que impactam a qualidade de vida dessa população, que, por sua vez, depende de políticas públicas integradas e efetivas.

Desenvolver redes sociais de apoio através de uma conduta interpessoal efetiva constitui um dos fatores mais importantes para se precaver e recuperar-se dos transtornos causados pelo envelhecimento. Estudos demonstram que o apoio recebido, não somente para a pessoa doente, mas também seu cuidador, torna-se um importante fator na qualidade de vida do binômio cuidador familiar e pessoa dependente, preservando a saúde de ambos (Bocchi \& Angelo, 2008; Brito \& Rabinovich, 2008).

Destaca-se como principal problema deste estudo o fato de que muitos dos benefícios, vantagens e contribuições da assistência domiciliar aparecem como consequência da cura e/ou reestabelecimento de uma patologia, deixando-se de lado o ser humano em sua totalidade e desconsiderando todas as suas dimensões, inclusive a espiritual.

Apesar dos poucos resultados encontrados, deve-se destacar o crescente número de serviços implantados no Brasil, o que demonstra não somente a relevância do tema, mas também sua ampliação para outras áreas, como a social e a da psicologia, proporcionando um avanço do conhecimento à medida que investiga os fatores envolvidos no processo de envelhecimento.

Recomendam-se outros estudos, com outros métodos de coleta de dados, bem como outro referencial a ser utilizado na análise dos dados coletados, e também com outros sujeitos de pesquisa que se envolvam no processo de cuidado na assistência domiciliar, principalmente àqueles cujo foco esteja em sua dinâmica social.

\section{REFERÊNCIAS}

Bardin, L. (2011). Análise de conteúdo. Lisboa: Persona.

Beger, M. M. (2008). Sistematização de um programa de treinamento da memória de pessoas idosas incorporando a autoavaliação. Tese de Doutorado, Faculdade de São Paulo, São Paulo.

Bocchi, S. C., \& Angelo, M. (2008). Between freedom and reclusion: social support as a quality-of-life component in the family caregiverdependent person binomial. Rev. Latino-Am. Enfermagem, 16(1), 15-23.

Bradbury-Jones, C., Irvine, F., Jones, C., Kakehashi, C., \& Ogi, A. (2011). A comparison of elderly care nursing in the UK and Japan. Nursing Older People Magazine. University of Dundee, 31-35.

Brito, E. S., \& Rabinovich, E. P. (2008). Desarrumou tudo! O impacto do Acidente Vascular Encefálico. Saude Soc. 17(2), 153-69.

Brondani, C. M. (2008). Desafios de cuidadores familiares no contexto da internação domiciliar. Dissertação de Mestrado, Universidade Federal de Santa Maria, Santa Maria, RS.

Doll, J. (2006). O campo interdisciplinar da Gerontologia. In: Py, L. et al. (Org.), Tempo de envelhecer. Percursos e dimensões psicossociais. São Paulo: Holambra.

Duarte, Y. A. O., \& Diogo, M. J. (2000). Atendimento domiciliário: um enfoque gerontológico. São Paulo: Atheneu.

Duayer, M. F. F., \& Oliveira, M. A. C. (2005). Cuidados domiciliários no SUS: uma resposta às necessidades sociais de saúde de pessoas com 
perdas funcionais e dependência. Revista Saúde em Debate, 29(70), 198-209.

Feuerwerker, L. C. M., \& Merhy, E. E. (2008). A contribuição da atenção domiciliar para a configuração de redes substitutivas de saúde: desinstitucionalização e transformação de práticas. Revista Panamericana de Salud Publica, v.24, 180-188.

Ganong, L. H. (1987). Integrative reviews of nursing research. Res Nurs Health, 10, 1-11.

Guedea, M. T. D., Albuquerque, F. J. B., Tróccoli, B. T., Noriega, J. A. V., Seabra, M. A. B., \& Guedea, R. L. D. (2006). Relação do bem estar subjetivo, estratégias de enfrentamento e apoio social em idosos. Psicol. Reflex. Crit., Porto Alegre, v.19, n.2, 301-308.

Hayashi, T., Nomura, H., Ina, K., Kato, T., Hirose, T., Nonogaki, Z., \& Suzuki, Y. (2011). Place of death for the elderly in need of end-of-life home care: a study in Japan. Archives of Gerontology and Geriatrics, 242-244.

Lacerda, M. R., Giacomozzi, C. M., Oliniski, S. R., \& Truppel, T, C. (2006). Atenção à saúde no domicílio: modalidades que fundamentam sua prática. Saúde e Sociedade, v.15, n.2, 88-95.

Lei n. 8.842 de 4 de janeiro de 1994. (1994, 4 de janeiro). Dispõe sobre a Política Nacional do Idoso, cria o Conselho Nacional do Idoso e dá outras providências. Brasília, DF: Secretaria Nacional dos Direitos Humanos.

Lupi-Pegurier, L., Clerc-Urmes, I., Abu-Zaineh, M., Paraponaris, A., \& Ventelou, B. (2011). Density of dental practitioners and access to dental care for the elderly: a multilevel analysis with a view on socio-economic inequality. Health Policy, 160-167.

Medeiros, F. A. L., Nóbrega, M. M. L., Sousa, F. S., \& Silva, G. S. (2011). Discurso de acompanhantes hospitalares sobre o cuidar de idosos: subsídio para uma orientação aos cuidados domiciliares pós-alta. Nursing (SP), 496500 .

Oliveira, V. C. R., Nogueira, L. S., Andolhe, R., Padilha, K. G., \& Sousa, R. M. C. (2011). Clinical evolution of adult, elderly and very elderly patients admitted in Intensive Care Units. Revista Latino Americana de Enfermagem, 19(6), 1344-1351.

Patrício, K. P. (2009). Ressignificação existencial do pretérito e longevidade humana. Saúde Soc. São Paulo. 18(2), 273-283.

Pérodeau, G., Paradis, I., Grenier, S., O'Connor, K., \& Grenon, E. (2011). Chronic psychotropic drug use among frail elderly women receiving home care services. Journal of Women \& Aging, 23(4), 321-341.
Portaria n. 2.528 de 19 de outubro de 2006. (2006, 19 de outubro). Aprova a Política Nacional de Saúde da Pessoa Idosa. Brasília, DF: Ministério da Saúde.

Resende, M. C., Cunha, C. P. B., Silva, A. P., \& Sousa, S. J. (2007). Rede de relações e satisfação com a vida em pessoas com amputação de membros. Ciências \& Cognição, Rio de Janeiro, v.10, 164-177.

Rodrigues, M. A., \& Seidl, E. M. F. (2008). A importância do apoio social em pacientes coronarianos. Paideia, 18(40), 279-288.

Sanya, E. O., Abiodun, A. A., Kolo, P., Olanrewaju, T. O., \& Adekeye, K. (2011). Profile and causes of mortality among elderly patients seen in a tertiary care hospital in Nigeria. Annals of African Medicine, 10(4), 278-283.

Sarton, G. (1998). Medicine in Old Egypt. In: History of Science. Heidelberg: Hamed A. Ead.

Schwonke, C. R. G. B., Silva, J. R. S., Casalinho, A. L. D., Santos, M. C., \& Vieira, F. P. (2008). Internação domiciliar: reflexos sobre a participação do cuidador/família/enfermeiro no cuidado. Ensaios e Ciência: Ciências Biológicas, Agrárias e da Saúde, v.12, n.1, 77-90.

Silva, R. R., \& Fonseca, T. M. A. (2007). Proteção social: notas críticas sobre a interface entre Estado e família na contemporaneidade [CDROM]. In Jornada Internacional em Políticas Públicas, 3. Maranhão, UFMA.

Siqueira, M. M. M. (2008). Construção e validação da escala de percepção de suporte social. Psicologia em Estudo, Maringá, v.13, n.2, 381-388.

Tamanini, J. T., Santos, J. L., Lebrão, M. L., Duarte, Y. A., \& Laurenti, R. (2011). Association between urinary incontinence in elderly patients and caregiver burden in the city of São Paulo/Brazil: Health, Wellbeing, and Ageing Study. Neurourology and Urodynamics, 30(7), 1281-1285.

Veras, R. (2009). Envelhecimento populacional contemporâneo: demandas, desafios e inovações. Revista Saúde Pública, São Paulo, v.43, n.3, 548-554.

Recebido em 23/07/2012 Aceito em 18/12/2012

Andreza Aparecida de Lima: psicóloga, mestre em Saúde Coletiva pela Faculdade de Medicina da Universidade Estadual Paulista Júlio de Mesquita Filho, Botucatu-SP, Brasil.

Regina Stella Spagnuolo: enfermeira, doutora em Saúde Coletiva, professora assistente do Departamento de Enfermagem da Universidade Estadual Paulista Júlio de Mesquita Filho, Botucatu-SP, Brasil.

Karina Pavão Patrício: médica, doutora em Saúde Pública, professora assistente da Faculdade de Medicina da Universidade Estadual Paulista Júlio de Mesquita Filho, Botucatu-SP, Brasil. 